\title{
Biocomputational prediction of non-coding RNAs in model
} cyanobacteria

\author{
Björn Voß ${ }^{1}$, Jens Georg1, Verena Schön ${ }^{1}$, Susanne Ude ${ }^{1}$ and \\ Wolfgang R Hess*1,2
}

\author{
Address: ${ }^{1}$ University of Freiburg, Faculty of Biology, Genetics and Experimental Bioinformatics, Schänzlestr. 1, D-79104 Freiburg, Germany and \\ ${ }^{2}$ Freiburg Initiative in Systems Biology, Schänzlestr. 1, D-79104 Freiburg, Germany \\ Email: Björn Voß - bjoern.voss@biologie.uni-freiburg.de; Jens Georg - jens.georg@biologie.uni-freiburg.de; \\ Verena Schön - schoen.verena@web.de; Susanne Ude - Susanne.Ude@gmx.de; Wolfgang R Hess* - wolfgang.hess@biologie.uni-freiburg.de \\ * Corresponding author
}

Published: 23 March 2009

BMC Genomics 2009, I0:123 doi:10.1186/147I-2164-10-123

This article is available from: http://www.biomedcentral.com/147I-2/64/10/123

(C) 2009 Voß et al; licensee BioMed Central Ltd.

This is an Open Access article distributed under the terms of the Creative Commons Attribution License (http://creativecommons.org/licenses/by/2.0), which permits unrestricted use, distribution, and reproduction in any medium, provided the original work is properly cited.
Received: 29 August 2008

Accepted: 23 March 2009

\begin{abstract}
Background: In bacteria, non-coding RNAs (ncRNA) are crucial regulators of gene expression, controlling various stress responses, virulence, and motility. Previous work revealed a relatively high number of ncRNAs in some marine cyanobacteria. However, for efficient genetic and biochemical analysis it would be desirable to identify a set of ncRNA candidate genes in model cyanobacteria that are easy to manipulate and for which extended mutant, transcriptomic and proteomic data sets are available.

Results: Here we have used comparative genome analysis for the biocomputational prediction of ncRNA genes and other sequence/structure-conserved elements in intergenic regions of the three unicellular model cyanobacteria Synechocystis PCC6803, Synechococcus elongatus PCC630I and Thermosynechococcus elongatus BPI plus the toxic Microcystis aeruginosa NIES843. The unfiltered numbers of predicted elements in these strains is $383,168,168$, and 809 , respectively, combined into 443 sequence clusters, whereas the numbers of individual elements with high support are $94,56,64$, and 406, respectively. Removing also transposon-associated repeats, finally 78,53, 42 and I 68 sequences, respectively, are left belonging to 109 different clusters in the data set. Experimental analysis of selected ncRNA candidates in Synechocystis PCC6803 validated new ncRNAs originating from the fabF-hoxH and apcC-prmA intergenic spacers and three highly expressed ncRNAs belonging to the Yfr2 family of ncRNAs. Yfr2a promoter-luxAB fusions confirmed a very strong activity of this promoter and indicated a stimulation of expression if the cultures were exposed to elevated light intensities.

Conclusion: Comparison to entries in Rfam and experimental testing of selected ncRNA candidates in Synechocystis PCC6803 indicate a high reliability of the current prediction, despite some contamination by the high number of repetitive sequences in some of these species. In particular, we identified in the four species altogether 8 new ncRNA homologs belonging to the Yfr2 family of ncRNAs. Modelling of RNA secondary structures indicated two conserved single-stranded sequence motifs that might be involved in RNA-protein interactions or in the recognition of target RNAs. Since our analysis has been restricted to find ncRNA candidates with a reasonable high degree of conservation among these four cyanobacteria, there might be many more, requiring direct experimental approaches for their identification.
\end{abstract}




\section{Background}

In bacteria, non-coding RNAs (ncRNAs) are a heterogeneous group of sequence-specific regulators of gene expression, normally lacking a protein-coding function. They are typically 50-250 nucleotides in length [1], and regulate mRNA translation or decay but sometimes also directly modulate certain protein functions. Most stress responses in the organism best-studied in this respect, E. coli, include at least one small regulatory RNA as part of the regulon [2]. However, their functions also include the control of plasmid and viral replication [3], bacterial virulence [4], quorum sensing [5], or the acquired resistance against bacteriophages [6].

In many cases, these ncRNAs function through sequencespecific base pairing; hence they frequently have a (partial) base complementarity to their target RNA molecules. The vast majority of known ncRNAs is encoded at genomic locations far away from their target genes. However, some ncRNAs are transcribed from the reverse complementary strand of the respective target and hence these are fully or partially overlapping with their target RNAs, constituting the class of antisense RNAs. Except for the more common types of ncRNA (ribosomal RNA, tRNA, tmRNA, 6S RNA, RNAse P RNA and ffs RNA), genes encoding ncRNAs are not annotated during standard genome analysis. The efforts to accomplish their identification in bacteria can broadly be divided into (i) sequencing the population of small RNAs or (ii) prediction by bioinformatics tools (mostly) followed by experimental verification (see [7] for review). As a result of such systematic searches, more than 80 ncRNAs are now known in $E$. coli, most of which had been overlooked by traditional genome analysis.

Cyanobacteria currently raise considerable interest as they perform oxygenic photosynthesis, fix atmospheric $\mathrm{CO}_{2}$ and nitrogen, frequently produce large quantities of bioactive secondary metabolites and due to their potential for the production of biofuels. As long as there is sufficient light available for photosynthesis, cyanobacteria populate widely diverse environments such as freshwater, the oceans, rock surfaces, desert soil or the polar regions. Their adaptation to vastly different environmental conditions suggests the existence of sophisticated regulatory mechanisms. Therefore, various types of regulatory RNA can be expected that interplay with the different signal transduction pathways and stress responses. Indeed, computational-experimental screens based on comparative genome analysis identified seven different ncRNAs in the marine cyanobacteria Prochlorococcus and Synechococcus [8] which were called Yfr1-7 for cY Yanobacterial Functional RNA. In a follow-up study making use of high density microarrays and exploiting the genome information from meanwhile 12 different Prochlorococcus genome sequences, additional 14 ncRNAs and 24 antisense RNAs were found [9]. Unicellular marine cyanobacteria of these genera provide an excellent dataset for computational predictions that require comparative genome information since currently 22 different genome sequences from very closely related isolates are available $[10,11]$. However, a major bottleneck in the work with these marine cyanobacteria is that despite some recent progress [12], protocols for genetic manipulation are very slow or not available at all. Therefore, the finding that two of these ncRNAs are phylogenetically widely distributed enabled direct genetic work on their functional relevance: Yfr1 is distributed throughout the cyanobacterial radiation [13] and might play a role in the adaptation to redox stress or the regulation of carbon uptake [14], whereas Yfr7 was identified as the homolog of the 6S RNA [15] which is found in all eubacteria [16]. However, for efficient genetic and biochemical analysis of cyanobacterial ncRNAs it would be very desirable to identify a set of ncRNA candidate genes in model cyanobacteria that are easy to manipulate and for which extended mutant, transcriptomic and proteomic data sets are available. In addition to Yfr1 which exists in all four unicellular cyanobacteria targeted here [13], the only currently known ncRNAs in model cyanobacteria are an antisense RNA covering the ferric uptake regulator gene furA in Anabaena PCC 7120 over its full length [17], and the antisense RNA IsrR, regulating the gene for the light-absorbing protein IsiA under conditions of iron limitation and redox stress in the unicellular Synechocystis PCC 6803 [18].

In recent years, comparative genomics-based prediction of ncRNA genes has become a standard method to search for such genes within bacterial genomes [8,19-23]. Thus, the availability of genome sequences from closely enough related species is a critical factor as is the conservation of ncRNAs. In case of unicellular cyanobacterial model organisms, the lack of genome sequences from close relatives has been hampering such studies. With the recent release of the Microcystis aeruginosa NIES-843 genome [24], however, a cyanobacterium relatively close to Synechocystis has been sequenced.

Here we set out to identify possible ncRNA genes and other RNA elements (5' leader sequences and riboswitches) in the three unicellular model cyanobacteria Synechocystis PCC6803, Synechococcus elongatus PCC6301 and Thermosynechococcus elongatus BP1 plus the toxic Microcystis aeruginosa NIES-843 (from now on: Synechocystis, Synechococcus, Thermosynechococcus and Microcystis) by biocomputational comparative genome analysis with a focus on Synechocystis.

\section{Results and Discussion Computational screening for novel ncRNAs}

To screen for novel RNA elements, all intergenic regions $>50$ nt were extracted from the four genomes and ana- 
lyzed as outlined in Fig. 1, leaving out all annotated genes, including tRNA and four structural RNA genes. Although single sequence elements and families of sequence elements that are specific to a single genome cannot be found by our approach, this procedure initially returned 443 predicted clusters holding 1528 individual sequences, from which 383 belonged to Synechocystis, 168 to Synechococcus, 168 to Thermosynechococcus and 809 to Microcystis. However, these numbers were diminished in further filtering steps (see below).

The analysis was basically focused on sequence and structure similarities. Detailed information on all clusters predicted by our method including the positions of all sequences is available online [25]. This information, which we show exemplarily in the inset in Fig. 1, includes the location within the compared genomes, flanking genes, a secondary structure prediction as well as Z-scores and probabilities in either forward $(Z, P)$ or reverse $(Z$ rev, $P$ rev) orientation as computed by RNAz [26]. Furthermore we conducted searches against Rfam [27], the database collecting ncRNAs, and TransTermHP [28], of which the results are also given in the online material.

\section{High-scoring putative RNA elements}

Filtering with $\mathrm{P}>=0.5$ or $\mathrm{Z}<=-2.0$ reduced the initial number of 1528 individual sequences in the 443 predicted clusters to 113 sequence clusters with 620 individ-

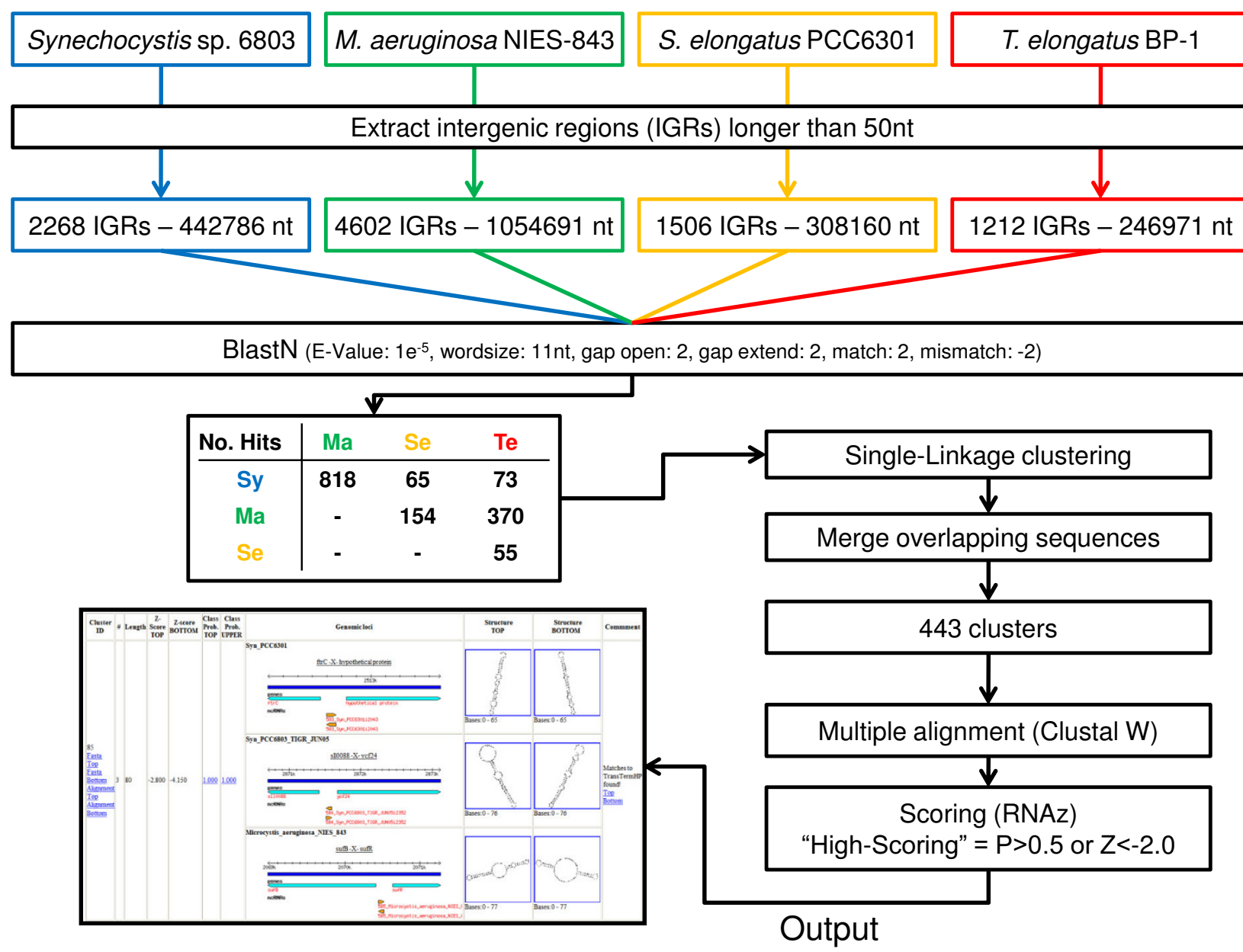

Figure I

Pipeline for comparative prediction of RNA elements and ncRNAs. Intergenic sequences of at least $50 \mathrm{nt}$ were gathered from four cyanobacterial genomes and locally aligned using BLASTN. Sequences which directly produced a significant blast hit $\left(E\right.$-value $\left.<10^{-5}\right)$ or which were connected by a chain of such hits were gathered into clusters ("single-linkage clustering"). After an additional unification step of overlapping sequences within each cluster the resulting clusters and their complement clusters were scored using RNAz [26]. The inset shows how this information is provided in the internet, together with the location within the compared genomes, using the top-scoring cluster (CLID 80) as an example. 
ual elements, 94, 56, 64 and 406 in Synechocystis, Synechococcus, Thermosynechococcus and Microcystis, respectively. A summary of the highest scoring clusters is given in Table 1[29-31]. The Venn diagram in Fig. 2[32] shows that more homologs were detected in the Synechocystis/ Microcystis comparison than in any other pairwise combination, reflecting the phylogenetic relationships between these species pairs.

We previously showed the existence of Yfr1 in three out of four tested marine cyanobacteria belonging to the genera Prochlorococcus and Synechococcus [8] and later demonstrated its existence throughout the cyanobacterial radiation, including the four unicellular cyanobacteria targeted here [13]. It was, therefore, no surprise to find Yfr1 among the top-scoring elements (Z-score and probability of 4.340 and 1.0) in cluster 139 (Table 1). Although RNA elements in cyanobacteria are only scarcely covered by Rfam, the existing entries provided another positive control set: the thiamine riboswitch was correctly identified in three strains (cluster 149; Table 1) and also two RNA elements of unknown function were correctly found for Synechocystis and Microcystis but not for the other two cyanobacteria (cluster 216 and 107 in Table 1). However, we noted the functional role assumed for one of these conserved RNA structures, the $\gamma k k C / \gamma x k D$ element (cluster 216), to switch efflux pumps and detoxification systems in response to harmful environmental molecules, may not apply to the cyanobacterial homologs since they are neither in Synechocystis nor in Microcystis located upstream of a putative transporter gene.

\section{Synteny among high-scoring RNA elements}

The genomic location of a predicted ncRNA gene or RNA element in the same sequence neighbourhood in some or all of the studied cyanobacteria can also be a powerful tool for finding related ncRNAs. Among the 25 high-scoring sequence clusters in Table 1, 9 (36\%) showed at least partial synteny. The high scoring element in cluster 80 illustrates this fact. The primary annotation gives no hint about the possible relatedness of the flanking genes. The flanking gene sufR annotated in Microcystis encodes an iron-sulfur cluster biosynthesis transcriptional regulator and similarity searches revealed that sll0088, syc2358d and sufR actually are orthologs of each other (Fig. 3A). Flanking the intergenic region with the predicted RNA element on the other side, genes $y c f 24$ and $s u f B$ are clearly homologs of each other, whereas $\mathrm{ftrC}$ in Synechococcus is not. Yet, $f t r C$ has been inserted in this genomic region as the proximate gene, syc2356_c, codes for the homolog of $s u f B$ and $y c f 24$. Thus, the synteny among neighbouring genes clearly support the element predicted in cluster 80 as an orthologous RNA element between the three species. Other cases of partial synteny in flanking genes are observed in cluster 139 since trxA is present in 3 out of 4 cases and in cluster 216 with the orthologs speB (Synechocystis) and agmatinase (Microcystis), whereas all other genes are different. Special cases of synteny are exposed in cluster 207 (rpl10 leader), 149 (thiamine riboswitch upstream of thiC), 394 (rps2 leader) and 62 (upstream groES). These four examples represent structurally conserved sequence elements upstream of a protein-coding gene to whom they are functionally connected; among them one riboswitch and two ribosomal leaders, thus this position must be conserved. The fourth example, the element upstream of groES contains the palindromic CIRCE element (Fig. 3B) thought to bind the heat-shock repressor protein HrcA [33]. Here, we mapped the groES transcriptional start site to the first nt of the nine nt loop predicted by secondary structure analysis (Fig. 3B), confirming the previously determined start site [34]. These examples illustrate the variety of elements that become identified by our approach.

\section{Experimental verification}

For exemplary experimental verification of predicted ncRNA genes we chose two very different examples, one well-supported candidate with three members from cluster 159 (probability 0.933 and Z-score -2.00; Table 1) and one from cluster 294 (probability 1.0 and Z-score -2.64; Table 1). Northern hybridization of total RNA from Synechocystis using strand-specific RNA probes confirmed the existence of both ncRNAs (Fig. 4). Since we verified the existence of both ncRNAs experimentally, we decided to name these two ncRNAs SyR1 and SyR2, for Synechocystis nct $N A \underline{1}$ and $\underline{\mathbf{2}}$. SyR1 is a strongly accumulating ncRNA transcribed from a gene in the fabF - hoxH IGR in the forward direction as the preceding gene fabF (Fig. 4A). The syr1 gene corresponds with a length of 130 nt to about two thirds of the fabF-hoxH intergenic spacer (length 206 nt). Judged by Northern hybridization, there was no evidence for a possible cotranscription with fabF. The element predicted with the CLID 294 is located 3' to a protein-coding gene, too, and is transcribed from the forward strand in Synechocystis 6803. SyR2 is an 140 nt ncRNA transcribed from a gene in the apcC (ssr3383) prmA (sll1909) IGR in the same forward direction as the preceding gene apcC. SyR2 is accumulated to rather high amounts, too, but these appeared lower than in case of SyR1 (Fig. 4B). The preceding apcC gene (ssr3383) encodes a short phycobilisome LC linker polypeptide and is the ultimate gene of a three-gene operon for phycobiliproteins. Cotranscription between this operon and SyR2 cannot be excluded unambiguously. However, a SyR2 transcript start was mapped within $a p c C, 49 \mathrm{nt}$ before the end of the reading frame. This fact is less exotic than it seems. At the expected spacing six nt upstream, the transcript start is preceded by a regular TATA element (CAAAAT). Moreover, several examples indicate the location of ncRNA promoters within the protein-coding part 
Table I: List of selected top-scoring RNA elements (see complete list at [25]).

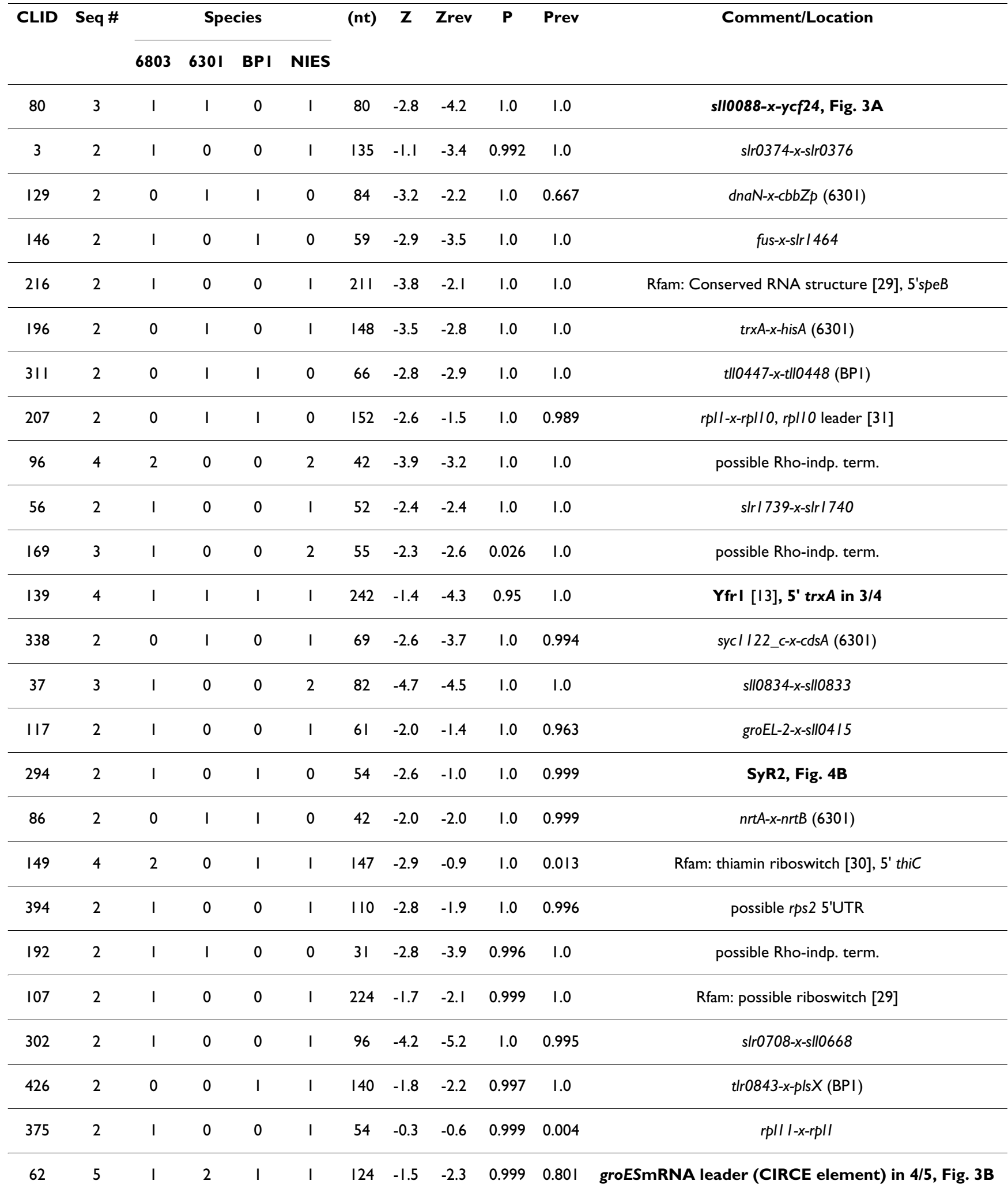

8 intermediate clusters! 
Table I: List of selected top-scoring RNA elements (see complete list at [25]). (Continued)

\begin{tabular}{|c|c|c|c|c|c|c|c|c|c|c|c|}
\hline \multicolumn{12}{|c|}{17 intermediate clusters! } \\
\hline 159 & 4 & I & I & 0 & 2 & 186 & -2.0 & -1.4 & 0.933 & 0.411 & SyRI, Fig. 4A \\
\hline
\end{tabular}

of a gene: Transcription of the ssrS gene for 6S RNA in $E$. coli is initiated at two promoters, from these the distally located promoter P2 responds to $\sigma 70$ and $\sigma S$ RNA polymerase holoenzymes and is located within the $y g f E$ reading frame $[35,36]$. An example from Synechocystis is provided with IsrR, the antisense RNA that is initiated from within the gene isiA, although from the reverse complementary strand [18].

In a more general sense these results demonstrate that, just judging from the prediction, both candidate ncRNA genes might have been expected to be 3'UTRs due to their close location to an mRNA 3 'end. We did not investigate their origin from a specific promoter further as we did for the Yfr2a ncRNA (see below), but the results shown in Fig. 4 , in particular the lack of a longer transcript signal in the respective agarose gel blots, plus specific RACE signals confirm unambiguously that they do accumulate as individual small transcripts and therefore constitute bona fide ncRNAs.



Figure 2

Venn diagram showing the numbers of predicted sequence clusters that receive high support $(P>0.5$ and/or $Z$ score $<-2.0$ ) and their distribution along the different genome combinations. The maximum number of 46 elements is shared between Synechocystis and Microcystis and only six are present in all genomes. One of these is Yfr I, other examples are CIRCE (Fig. 3) and the Yfr2 ncRNA family (Fig. 5). The figure was produced using Venny [32].

\section{A family of ncRNAs that is widely conserved among cyanobacteria}

The vast majority of the 100 bacterial ncRNAs experimentally verified thus far have been identified in Escherichia coli [2] and a few other model proteobacteria and Pseudomonas species. Therefore it is not surprising that, with the exception of the four highly conserved ncRNAs 6S RNA, tmRNA, ffs and RnpB, ortholog genes for ncRNAs are known only among very closely related species such as between Salmonella sp. and Yersinia sp. [37]. Here, with cluster 219 eight sequences were identified with high sequence and predicted secondary structure similarity to a family of ncRNAs initially found in marine Prochlorococcus [8]. There are four such ncRNAs in Prochlorococcus MED4 which in the original publication had been named Yfr2, Yfr3, Yfr4 and Yfr5 [8]. From the eight new members to this family in cluster 219 three belong to Synechocystis, one to Thermosynechococcus and two each are predicted in Synechococcus and in Microcystis. Since none of them has a more pronounced similarity to any of the original Yfr2-Yfr5 ncRNAs from Prochlorococcus MED4, we decided to call them all "Yfr2" according to the first member in this group and then just to add a suffix. Therefore, the three predicted candidates belonging to this ncRNA family in Synechocystis are Yfr2a, Yfr2b and Yfr2c. All three are expressed in Synechocystis (Fig. 5). The yfr2a gene is located downstream of thioredoxin A (trxA) gene sll1980. Both genes are in the same orientation but Yfr2a originates from a specific initiation site of transcription, mapped by TAP-RACE to position 1558975 in the genome (complementary strand), $94 \mathrm{nt} 3$ ' of the sll1980 stop codon (Fig. 5). The other two ncRNAs belonging to this family in Synechocystis, Yfr2b and Yfr2c, originate from genes directly upstream of two protein-coding genes, slr0199 and sll1477. In these two cases we mapped identical initiation sites of transcription for the ncRNAs and their respective downstream located protein-coding gene, to genomic positions 2730523 (forward strand, Yfr2b) and 3398352 (complementary strand, Yfr2c). Therefore, transcription of these two mRNAs occurs possibly by a read-through mechanism from Yfr2b or Yfr2c. Whether this type of transcriptional fusion has functional relevance is currently unknown as are the functions of slr0199 and sll1477. These genomic arrangements are not conserved as the genes adjacent to the other five candidate ncRNA genes belonging to this family differ in the other three 


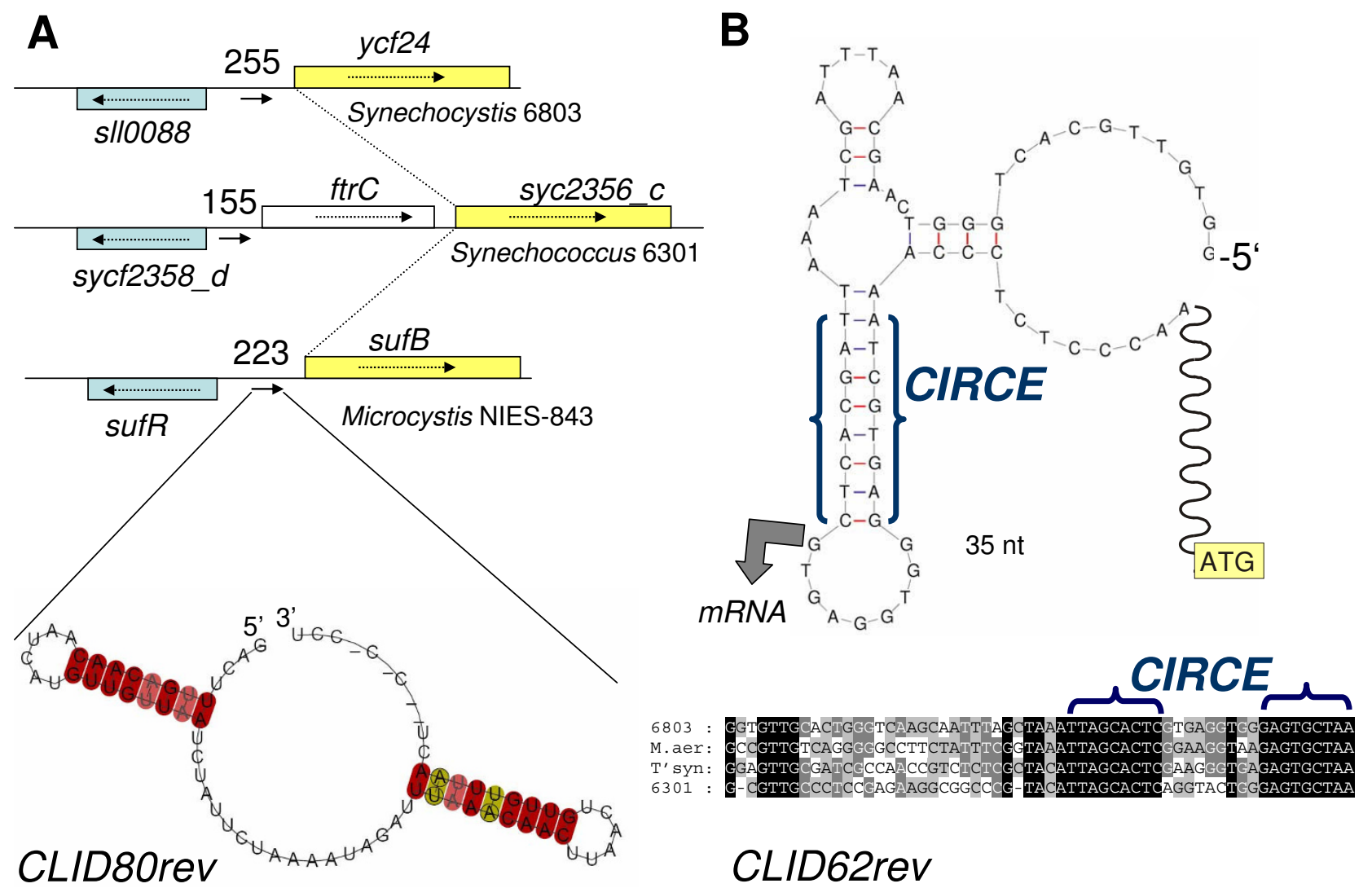

\section{Figure 3}

Types of predicted elements. A. The genomic location of predicted RNA element in cluster 80 and synteny around this element is shown. This element is slightly more likely to be transcribed from the forward strand as indicated by the direction of the arrow within the IGR. The length of the intergenic spacer is given in nt and homologous genes are colour-coded. In Synechococcus 630 I an ftrC gene has been inserted into this region relative to the other. The predicted consensus structure of the RNA element (bottom) consists of two stem-loops separated by a $17 \mathrm{nt}$ single-stranded region. The degree of sequence conservation is colour-coded. B. Four of the five sequences in cluster 62 are located upstream of the groES operon. This region is known to contain the palindromic CIRCE element and indeed, this element constitutes a critical part of the conserved sequence and structure. The initiation site of transcription of the groES mRNA was mapped by 5' RACE to occur from the first $\mathrm{G}$ within the nine $\mathrm{nt}$ loop that is part of the CIRCE element (bold arrow). The fifth sequence has no CIRCE element but has been clustered into cluster 62 based on other sequence features. At the bottom right, the perfect conservation of the CIRCE element in the four compared cyanobacteria is shown.

cyanobacteria. This lack of synteny supports a function of Yfr2b and Yfr2c independent from serving merely as 5' untranslated leaders of slr0199 and sll1477.

Sequence alignments and secondary structure predictions of the 8 Yfr2-5-type ncRNAs suggest a centrally located single-stranded loop element together with a short unpaired region at the $5^{\prime}$ end that are highly conserved (Fig. 6). The long helical stem bearing the $12 \mathrm{nt}$ loop is very characteristically predicted in all sequences to be interrupted by at least one bulge at position -4 with regard to this loop (Fig. 6). Interestingly, this feature is shared with the Yfr2-Yfr5 ncRNAs from marine cyanobacteria [8]. Bulge motifs have been recognized in a wide range of
RNAs as key structural elements determining molecular recognition by other molecules [38]. Therefore, the conserved bulges in Yfr2-type ncRNAs may indicate their interaction with proteins. Indeed, another hint comes from the unpaired regions of these ncRNAs which resemble the extended "GGA" and "ANGGA" RsmA-binding motifs. The ncRNAs RsmX, RsmY and RsmZ found in Pseudomonas species contain several GGA and extended ANGGA motifs [39]. For RsmY, these motifs have been shown to be essential for sequestration of RsmA and its homolog RsmE in Pseudomonas fluorescens [40]. Non-coding RNAs containing this motif frequently have a titrating role on their target protein, regulating gene expression at 

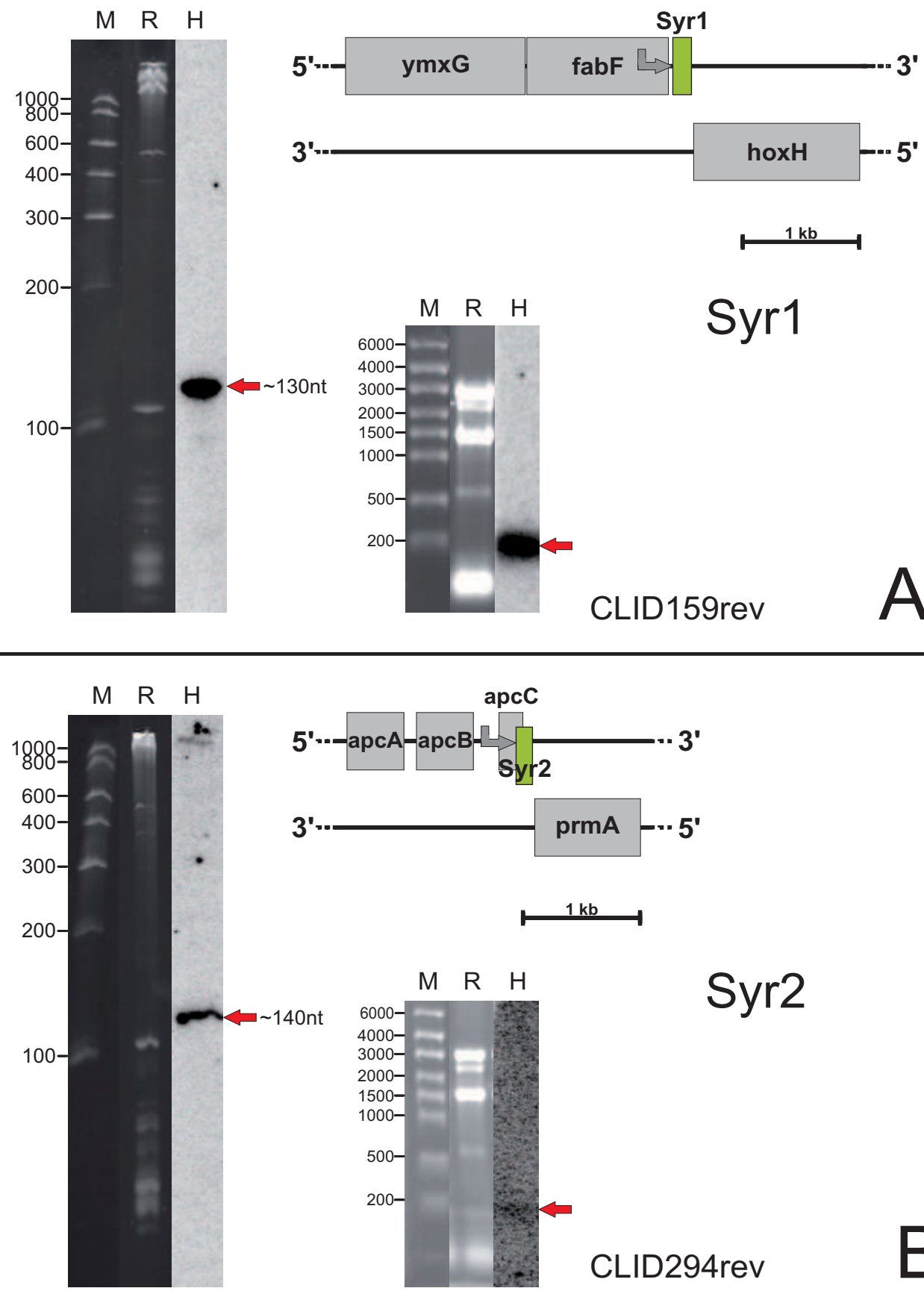

\section{Figure 4}

Experimental verification of two differently scoring ncRNAs by Northern hybridization. A. The element predicted in cluster 159 is transcribed from the forward strand (green), in the same direction as the preceding fabF (slr|332) gene. Six 5'RACE sequences support the transcript start of SyRI to be located $55 \mathrm{nt}$ 3' of the fabF reading frame at position 167/919 (grey arrow). Two different blots are shown, one in which RNA was separated in an high-resolution polyacrylamide gel and one resulting from an agarose gel. B. The element predicted with the CLID 294 was named SyR2. This ncRNA is longer than the IGR where it is encoded ( $\sim 40 \mathrm{nt}$ versus $94 \mathrm{nt}$ spacer length). A transcript start was found by 5' RACE within apcC, $49 \mathrm{nt}$ before the end of $a p c C$ (grey arrow). The schemes are drawn to scale. All protein-coding genes are displayed in gray, all ncRNA genes in green; $M$, molecular mass marker, $\mathrm{R}$, lane in the RNA gel before blotting, $\mathrm{H}$, hybridization. 


\section{Yfr2a}


$\stackrel{1 \mathrm{~kb}}{\longmapsto}$

\section{Yfr2b}
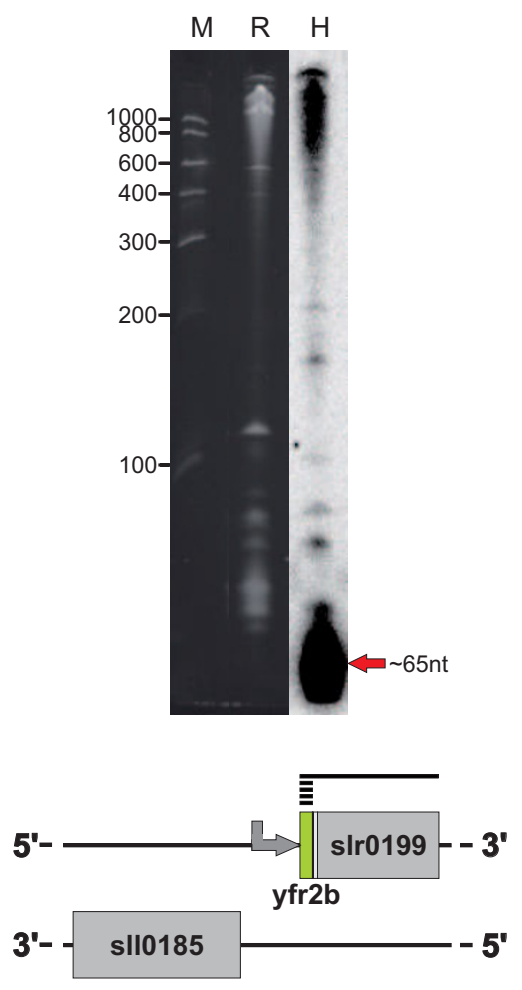

$1 \mathrm{~kb}$
Yfr2c
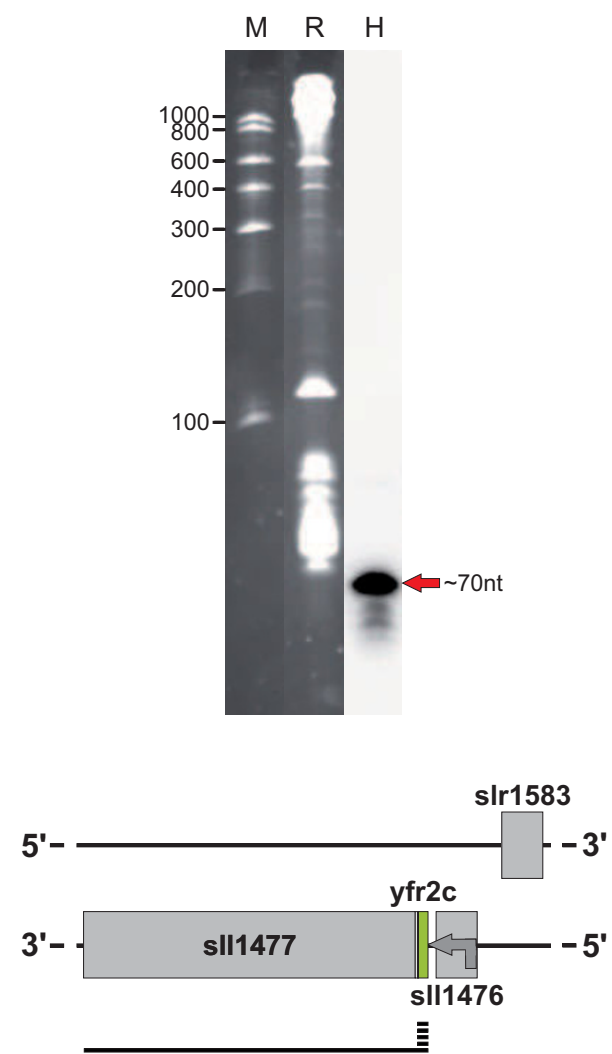

$1 \mathrm{~kb}$

\section{Figure 5}

All three predicted members of the Yfr2 family of ncRNAs are expressed in Synechocystis 6803 . The three predicted ncRNAs belonging to cluster 219 are expressed in Synechocystis 6803. The ncRNA Yfr2a is $\sim 80 \mathrm{nt}$ long. All initiation sites of transcription were mapped by 5' RACE (grey arrows). The transcriptional start site of Yfr2a was mapped unambiguously to position 1558975 (complementary strand), $93 \mathrm{nt}$ downstream the end of the trxA (sll I 980) open reading frame. Yfr2b and Yfr2c are initiated at positions 2730523 and 3398352 (complementary strand), respectively. All three ncRNAs are very strongly expressed in Synechocystis 6803. However, the fact that it was not possible to map a specific initiation site of transcription for the genes sIr0199 and sll I477 indicates their occasional or standard cotranscription with Yfr2b and Yfr2c. This assumption is further supported by some RNA fragments of higher molecular weight hybridizing with the Yfr $2 b$ probe. All labels are as in Fig. 4.

the translational level. It was not possible, however, to identify RsmA and RsmE homologs in cyanobacteria.

\section{Expression analysis of Yfr2a}

Starting with the mapped initiation site of Yfr2a we chose the region located immediately upstream of it in a promoter fusion experiment with $\operatorname{lux} A B$ genes to prove that it actually does contain a functional promoter. Moreover, if the expression of an ncRNA is regulated under certain environmental conditions this sometimes gives a hint into which processes this ncRNA might be involved in. As controls, we chose the same DNA fragment in reverse orientation and amplified and cloned the psbA2 (slr1311) promoter, again in both orientations.

The $300 \mathrm{nt}$ upstream of Yfr2a provided indeed very strong expression to the reporter genes - under all tested conditions the measured fluorescence values were comparable to those obtained from the $p s b A 2$ promoter-driven luciferase gene expression, whereas the reverse orientation of the same fragments provided very low activity only (Fig $7 \mathrm{~A})$. Under cold temperature $\left(12^{\circ} \mathrm{C}\right)$ and heat $\left(43^{\circ} \mathrm{C}\right)$ 
A


B



Figure 6

Sequence alignments and secondary structure predictions of the 8 Yfr2-type ncRNAs identify conserved structure and sequence motifs. A. Secondary structure predictions of the three experimentally confirmed ncRNAs Yfr2a, Yfr2b and Yfr2c from Synechocystis 6803. They share a $12 \mathrm{nt}$ central loop on a long helical stem that is interrupted by at least one bulge at position -4 with regard to this loop (red arrows). Moreover, the first 8-13 nt are predicted to be single-stranded. B. Alignments of all eight predicted Yfr2-type DNA sequences reveal two extremely conserved nucleotide stretches: the short unpaired region at the 5 ' end as well as the predicted centrally located loop element (labelled by horizontal black arrows). In contrast, the region between these two elements is not conserved in sequence or in its length. The single nucleotide breaking the stem at position -4 with regard to the loop is indicated by a red arrow. Note that the $3^{\prime}$ end of the transcribed region has not been mapped. Those sequence stretches resembling "GGA" and "ANGGA" motifs are labelled by a set of black arrows. The non-Synechocystis 6803 sequences are one from Thermosynechococcus (Thermo_Yfr2), two from Microcystis (Micro_Yfr2a and Micro_Yfr2b) and two from Synechococcus 630I (630I_Yfr2a and 630I_Yfr2b).

stress the activity of both promoters drops (Fig 7C, D), a possible pleiotropic effect. Under light stress, however, the activity of both promoters is stimulated. Whereas the activity of the $p s b A 2$ promoter is increased up to $250 \%$, the Yfr2a promoter becomes activated up to $300 \%$ (Fig $7 \mathrm{~B})$. This is all the more striking since $p s b A 2$ frequently serves as an example for a typical light-inducible promoter.

\section{Six Clusters containing repetitive sequences}

One problem when dealing with genome sequences of some cyanobacteria is the high number of repetitive 
A

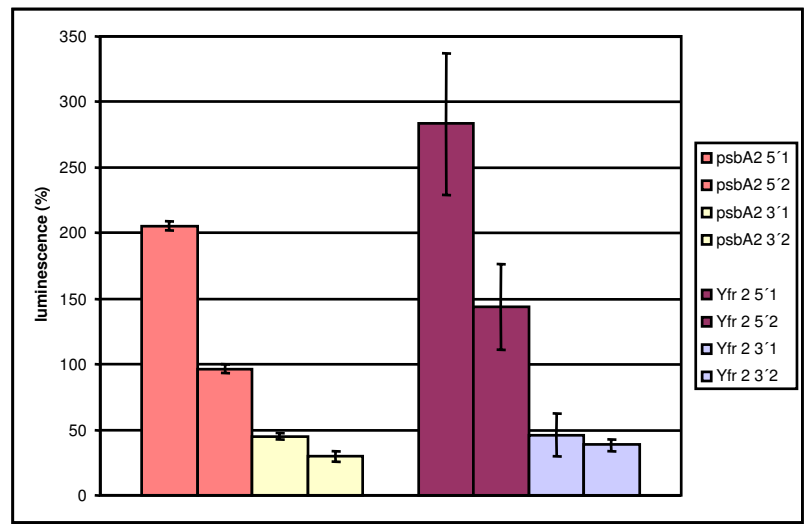

C

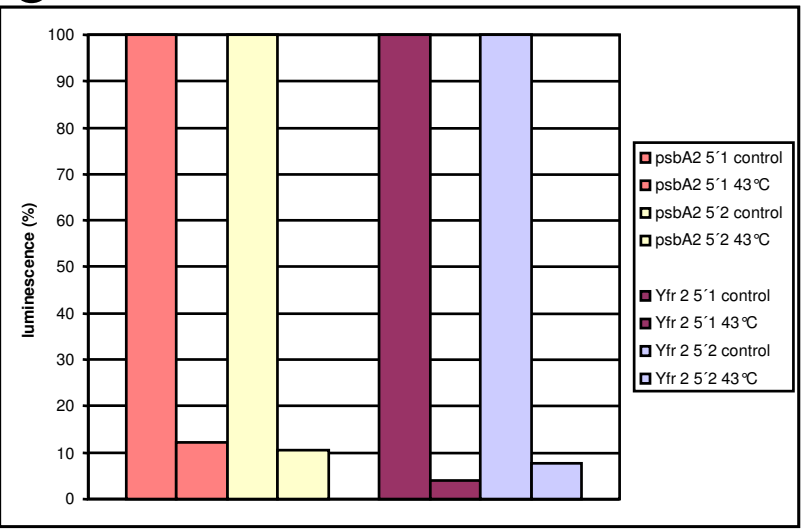

B

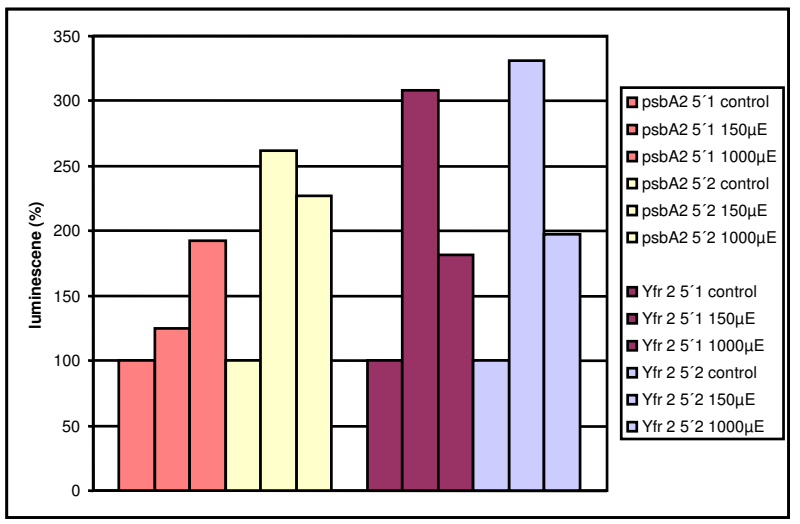

D

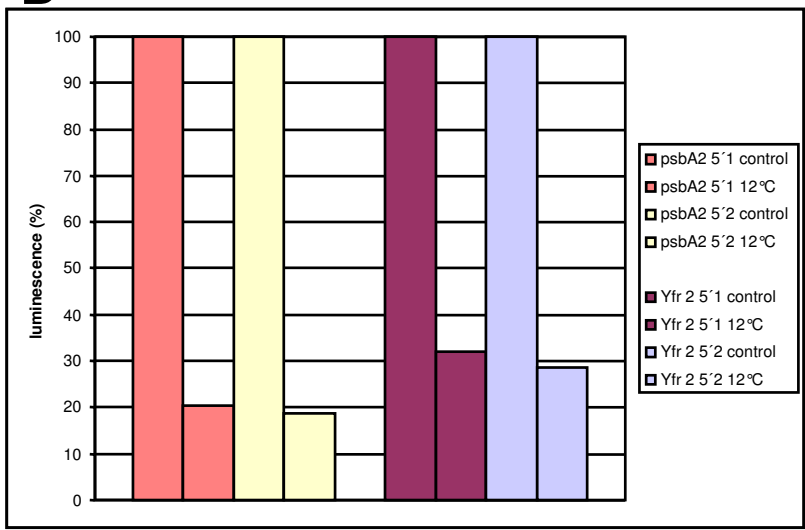

Figure 7

Activity of the Synechocystis Yfr2a promoter under various conditions. A. Fluorescence emitted from cultures with luciferase expression driven by the Yfr2a or the psbA2 (slrl3II) promoter under standard growth conditions. All promoter fragments were used in the correct (5') or inverted (3') orientation with two independent clones each. Average absolute luminescence values together with standard deviations from three replicate measurements for each clone are shown. B. Measurement of luciferase activity $20 \mathrm{~min}$ after application of two different light treatments, transfer of cultures to I50 and I,000 $\mu$ mol photons $\mathrm{s}^{-1} \mathrm{~m}^{-2}$ for 15 minutes. C. Heat stress; expression was measured 20 min after exposure to $43^{\circ} \mathrm{C}$ for 10 min. D. Cold temperature stress; expression was measured 20 min after exposure to $12^{\circ} \mathrm{C}$ for 10 min. All measurements were performed with two independent clones in three replicates each. In B, C and D the values from the untreated controls were set to $100 \%$. All cultures grew in late logarithmic phase during the measurements.

sequences, mobile genetic elements and transposonrelated sequences.

Indeed, the output from our prediction pipeline was contaminated by imperfect inverted repeat sequences flanking different families of IS elements, mainly in Microcystis, and to a lesser extent in Synechocystis. If subtracted from the data set, the total number of predicted RNA elements (numbers in brackets correspond to high-scoring elements) in Synechocystis, Synechococcus, Thermosynechococcus and Microcystis drops to 339 (78), 160 (53), 144 (42) and 426 (168). Such repetitive sequences were collected in six sequence clusters, namely \#12, \#25, \#38, \#68, \#270 and $\# 383$ with $78,87,153,83,42$ and 16 individual sequences, respectively. All 459 sequences from these clusters have been collected in a separate file and are accessible from our website [41].

\section{Conclusion}

Comparative genomics-based prediction of ncRNA genes and candidate ncRNA genes is more and more becoming a standard tool to search for such genes within bacterial genomes [8,19-23].

Here we provide the first list of ncRNA and other RNA element candidates for model unicellular cyanobacteria. Surprisingly, we identified with Yfr2a-Yfr2c a family of 
ncRNAs which is widely conserved among cyanobacteria and which become accumulated to high concentrations.

Our experimental verification together with existing positive controls suggests a high number of positives in this candidate set. However, there are also putative 5 ' operon leaders, Rho-independent 3' transcriptional terminators and possibly yet unidentified riboswitches in this data set. Moreover, the output is contaminated to some extent by transposase-related sequences.

Nevertheless, by analogy to other bacteria, including the most streamlined marine cyanobacterium Prochlorococcus MED4 [9], this number of ncRNAs and other RNA elements is probably a grave underestimation. Therefore this analysis should be considered as a first step to become complemented by more exhaustive experimental screens, for instance by using tiling arrays or deep sequencing in the near future.

\section{Methods}

\section{Cultures and manipulation of cyanobacteria}

The Synechocystis Moscow strain was used in this study (obtained from A. Wilde, University of Giessen, Germany, originally from S. Shestakov, Moscow State University, Russia) and propagated on BG11 [42] 1\% (w/v) agar (Bacto agar, Difco) plates. Liquid cultures of Synechocystis were grown at $30^{\circ} \mathrm{C}$ in BG11 medium under continuous illumination with white light of $50 \mu \mathrm{mol}$ of photons $\bullet \mathrm{m}$ $2 \bullet$ s- 1 and a continuous stream of air.

As a promoter test vector we used the pILA plasmid [43] into which 300 bp long promoter fragments were cloned as transcriptional fusions with the $\operatorname{lu} A B$ genes. After transformation, this plasmid integrates into the slr0168 gene within the chromosome of Synechocystis by homologous recombination. Transformation and analysis of correct integration and segregation was carried out as described elsewhere [44].

\section{Extraction and analysis of RNA}

Exponentially growing Synechocystis cultures (OD750 0,6 $-0,8$ ) were collected by filtration (Pall Supor 800 Filter). Filters with cells were dissolved in $1 \mathrm{ml}$ Trizol per $40 \mathrm{ml}$ culture, immediately frozen in liquid nitrogen and incubated for $15 \mathrm{~min}$ at $65^{\circ} \mathrm{C}$ in a water bath. Further RNA isolation followed the manufacturer's protocol.

Small RNA Northern blots were prepared from the separation of 10 to $25 \mu \mathrm{g}$ of total RNA on $10 \%$ urea-polyacrylamide gels as described by Steglich et al. [9]. Polyacrylamide gels were stained with ethidium bromide $(0.3 \mu \mathrm{g} / \mathrm{l})$ in $1 \times$ TBE buffer, rinsed with $1 \times$ TBE and analyzed with an E-BOX video gel documentation system (Peqlab). Transcript sizes were determined by correlation to Fermentas' RiboRuler low range RNA marker. Blots for RNAs with higher molecular weight were prepared from the separation of $5 \mu \mathrm{g}$ of total RNA on 1,5\% denaturing agarose gels. Transcriptional start sites were determined by 5 '-RACE as described [9].

\section{Sequence data}

Genome sequences were obtained from the finished microbial genomes website at Genbank [45] with the following accession numbers: Synechococcus elongatus PCC 6301, NC_006576; Synechocystis sp. PCC 6803, NC_000911; Thermosynechococcus elongatus BP-1, NC_004113; Microcystis aeruginosa NIES843, NC_010296.

\section{Prediction of RNA elements in a comparative approach}

We performed a comparative prediction of ncRNA elements within intergenic regions (IGRs). Therefore, all IGRs longer than $50 \mathrm{nt}$ were extracted and compared among the different genomes using Blast. Intragenomic analyses with the settings given in Fig. 1 revealed a high number of repetitive sequences in some of the analyzed genomes. There were 895 intragenomic hits within the genome of Synechococcus, 2227 hits within Thermosynechococcus, 7198 in Synechocystis and 557014 mutual hits of IGRs from Microcystis with other IGRs from the same organism. A large number of repetitive elements $\mathrm{N}$ would produce approximately $\mathrm{N}^{2}$ hits, meaning that the square root of the number of hits gives an estimate of the number of repetitive elements. In the case of Microcystis this yields 746 repetitive elements, which is supported by the finding of six large clusters holding 383 sequences from Microcystis. Therefore, we refrained from searching for intragenomic similarity. Based on these results homologous sequence regions got clustered together, aligned using ClustalW and analyzed for structural significance by RNAz. Alignments were postprocessed using the tool rnazSelectSeqs.pl with default parameters from the RNAz package. RNAz was applied in a sliding window approach (a step size of $10 \mathrm{nt}$ and different window sizes were used, namely 80,100,120,140 and $160 \mathrm{nt}$ ) of which the window with highest probability was selected. Elements are termed "High-scoring" if they achieve an RNAz probability of 0.5 or more or if their Z-score is -2.0 or below. Details about the individual steps and their outcome are shown in Fig. 1. All predictions can be found at [25] and [41].

\section{Matching predictions to Rfam and TransTermHP}

All individual sequences were matched against Rfam [27] using the batch search feature provided by Rfam. Mapping of predicted sequences to information about Rho-independent terminators provided by TransTermHP [28] was done using Vmatch [46]. Therefore, TransTermHP predictions for Microcystis were computed using TransTermHP 2.06 with default parameters, while for Synechocystis, Syn- 
echococcus and Thermosynechococcus existing predictions were downloaded from the TransTermHP website. All predictions were converted to FASTA-format and searched for at least $30 \mathrm{nt}$ long hits with 100\% identity to candidate sequences.

\section{Comparative sequence/structure analysis}

Multiple sequence alignments were generated using ClustalW [47] with default parameters for DNA. Comparative structure prediction was done with RNAlishapes [48], a tool which predicts a consensus structure for a set of aligned sequences by taking covariance and free energy into account. The resulting consensus structure was analysed together with the multiple sequence alignment using RALEE [49]. The latter served also for manual optimisation of the alignment and the consensus structure, respectively, and for the production of colour annotated alignments. Colour plots of Consensus structures were generated using RNAalifold [50].

\section{Oligonucleotides}

Oligonucleotide primers for the generation of hybridization probes (T7 promoter sequence in boldface letters):

Yfr2c_for: 5'-TAATACGACTCACTATAGGccgccagcgccattgcttc-3'

Yfr2c_rev: 5'-cttaggacaggtgtgaggaaattag-3'

Yfr2b_for: 5'-TAATACGACTCACTATAGGcggggagcatagaccagcttg-3'

Yfr2b_rev: 5'-ggaagttattatctagaggtgtgtgag-3'

Yfr2a_for: 5'-TAATACGACTCACTATAGGaggcaaaaaaataaggaagtccgcaag-3'

Yfr2a_rev: 5'-cggctatcccgccttagg-3'

Syr1_for: 5'-TAATACGACTCACTATAGGccgagggcatatctaggagaac-3'

Syr1_rev: 5'-ggctatggaaaccogacagaattc-3'

Syr2_for: 5'-TAATACGACTCACTATAGGcaaacaaaaaaagaggccattgctgacc-3'

Syr2_rev: 5'-gactagttgttgctaatttagcaatgttg-3'

Oligonucleotides for promoter fusion experiments (KpnI restriction sites 5'-GGTACC-3' introduced for cloning are labelled in boldface letters):

Yfr2a (fw): 5'-GGTACCCTAGATGACACCGGCACG-3'
Yfr2a (rev): 5'-GGTACCCTCCTCACACACAAATAAATGTTAG-3'

psbA2 (fw): 5'-CCTTGGTACCAAGAGTAATGGCGTGC-3'

psbA2 (rev): 5'-GATTGGTACCGGAACTGACTAAACTTAGTC-3'

Oligonucleotides for specific mapping of 5'ends through 5'RACE:

Yfr2c_5'RT: 5'-CCTAAAAATTGCCATAAAAAAACAC-3'

Yfr2c_5'Race: 5'-TCTTTCCTTGTTTCGACTCCAG-3'

sll1477_5'RT: 5'-GCGGCCAGAGGTTTCC-3'

sll1477_5'Race: 5'-CAGCGTAGCTAGGGAAATCACCACCAG-3'

Yfr2b_5'RT: 5'-AAAAGGCAAGAAAAAGCCCC-3'

Yfr2b_5'Race: 5'-TTTCCTTGTTTCGACTCCGGGG-3'

slr0199_5'RT: 5'-TGACCCAGATACCCTAAAAG-3'

slr0199_5'Race: 5'-CTTTTGATAATCTTGGCGGCC-3'

Yfr2a_5'RT: 5'-GGAGTCTTGCCATGTTTCG-3'

Yfr2a_5'Race: 5'-CCTCCGGCTGCTTCCTT-3'

\section{Hybridization conditions}

Northern hybridization was performed at $62^{\circ} \mathrm{C}$ in hybridization buffer (50\% deionized formamide, 7\% SDS, 250 $\mathrm{mM} \mathrm{NaCl}, 120 \mathrm{mM} \mathrm{Na}\left(\mathrm{PO}_{4}\right), \mathrm{pH}$ 7.2) as described by Steglich et al. [9]. Single stranded probes were generated from PCR-amplified templates incorporating the T7 promoter in one of the oligonucleotide primers, using the MAXIscript Kit (Ambion, USA) and 100 ng PCR-generated DNA template.

Detailed information on all clusters predicted by comparative genome analysis including the positions of all sequences can be found at: [25] and [41].

\section{Abbreviations}

IGR: intergenic region; ncRNA: non-coding RNA.

\section{Authors' contributions}

BV designed and carried out bioinformatic analyses and participated in drafting the manuscript, JG performed all RNA analyses in the laboratory, SU and VS constructed the promoter test constructs and performed the reporter gene 
assays. WRH designed research and wrote the manuscript. All authors read and approved the final manuscript.

\section{Acknowledgements}

This work was supported by the Deutsche Forschungsgemeinschaft Focus program "Sensory and regulatory RNAs in Prokaryotes" SPPI 258 (project HE 2544/4-I), the graduate school "Signal systems in plant model organisms" (to JG) and by the Freiburg Initiative in Systems Biology. We thank Martin Hagemann for the gift of promoter test vectors.

\section{References}

I. Vogel J, Papenfort K: Small non-coding RNAs and the bacterial outer membrane. Curr Opin Microbiol 2006, 9(6):605-6II.

2. Gottesman S: Micros for microbes: non-coding regulatory RNAs in bacteria. Trends Genet 2005, 21:399-404.

3. Wagner EG, Simons RW: Antisense RNA control in bacteria, phages, and plasmids. Ann Rev Microbiol 1994, 48:71 3-742.

4. Sittka A, Pfeiffer V, Tedin K, Vogel J: The RNA chaperone Hfq is essential for the virulence of Salmonella typhimurium. Mol Microbiol 2007, 63(I): 193-217.

5. Lenz DH, Mok KC, Lilley BN, Kulkarni RV, Wingreen NS, Bassler BL: The small RNA chaperone $\mathrm{Hfq}$ and multiple small RNAs control quorum sensing in Vibrio harveyi and Vibrio cholerae. Cell 2004, I I 8:69-82.

6. Brouns SJ, Jore MM, Lundgren M, Westra ER, Slijkhuis RJ, Snijders AP Dickman MJ, Makarova KS, Koonin EV, Oost J van der: Small CRISPR RNAs guide antiviral defense in prokaryotes. Science 2008, 32 I (589 I):960-964.

7. Vogel J, Sharma CM: How to find small non-coding RNAs in bacteria. Biol Chem 2005, 386( ( 2): 1219-1238.

8. Axmann IM, Kensche P, Vogel J, Kohl S, Herzel H, Hess WR: Identification of cyanobacterial non-coding RNAs by comparative genome analysis. Genome Biol 2005, 6:R73.

9. Steglich C, Futschik ME, Lindell D, Voss B, Chisholm SW, Hess WR The challenge of regulation in a minimal phototroph: Noncoding RNAs in Prochlorococcus. PLOS Genet 2008 , 4(8): 1000173.

10. Dufresne A, Ostrowski M, Scanlan DJ, Garczarek L, Mazard S, Palenik BP, Paulsen IT, Tandeau de Marsac N, Wincker P, Dossat C, et al.: Unravelling the genomic mosaic of a ubiquitous genus of marine cyanobacteria. Genome Biol 2008, 9:R90.

II. Kettler GC, Martiny AC, Huang K, Zucker J, Coleman ML, Rodrigue S, Chen F, Lapidus A, Ferriera S, Johnson J, et al:: Patterns and implications of gene gain and loss in the evolution of Prochlorococcus. PLoS Genet 2007, 3( I 2):e23 I.

12. Tolonen AC, Liszt GB, Hess WR: Genetic manipulation of Prochlorococcus strain MIT93 13: Green Fluorescent Protein expression from an RSF 1010 plasmid and $\mathrm{Tn} 5$ transposition. Appl Environ Microbiol 2006, 72:7607-76I3.

13. Voss B, Gierga G, Axmann IM, Hess WR: A motif-based search identifies the ortholog of the small RNA Yfrl in all major lineages of cyanobacteria. BMC Genomics 2007, 8:375.

14. Nakamura T, Naito K, Yokota N, Sugita C, Sugita M: A cyanobacterial non-coding RNA, Yfr I, is required for growth under multiple stress conditions. Plant Cell Physiol 2007, 48: | 309-|3|8.

15. Axmann IM, Holtzendorff J, Voss B, Kensche P, Hess WR: Two distinct types of 6S RNA in Prochlorococcus. Gene 2007, 406:69-78.

16. Barrick JE, Sudarsan N, Weinberg Z, Ruzzo WL, Breaker RR: 6S RNA is a widespread regulator of eubacterial RNA polymerase that resembles an open promoter. RNA 2005, I I(5):774-784.

17. Hernandez JA, Muro-Pastor AM, Flores E, Bes MT, Peleato ML, Fillat MF: Identification of a furA cis antisense RNA in the cyanobacterium Anabaena sp. PCC 7 I 20 . I Mol Biol 2005, 355:325-334

18. Duehring U, Axmann IM, Hess WR, Wilde A: An internal antisense RNA regulates expression of the photosynthesis gene isiA. Proc Nat Acad Sci USA 2006, 103:7054-7058.

19. del Val C, Rivas E, Torres-Quesada O, Toro N, Jiménez-Zurdo Jl: Identification of differentially expressed small non-coding RNAs in the legume endosymbiont Sinorhizobium meliloti by comparative genomics. Mol Microbiol 2007, 66(5):1080-1091.
20. González N, Heeb S, Valverde C, Kay E, Reimmann C, Junier T, Haas $D$ : Genome-wide search reveals a novel GacA-regulated small RNA in Pseudomonas species. BMC Genomics 2008, 9:167.

21. Mandin P, Repoila F, Vergassola M, Geissmann T, Cossar P: Identification of new noncoding RNAs in Listeria monocytogenes and prediction of mRNA targets. Nucleic Acids Res 2007, 35:962-974.

22. Padalon-Brauch G, Hershberg R, Elgrably-Weiss M, Baruch K, Rosenshine I, Margalit H, Altuvia S: Small RNAs encoded within genetic islands of Salmonella typhimurium show host-induced expression and role in virulence. Nucleic Acids Res 2008, 36(6): $1913-1927$

23. Ulvé VM, Sevin EW, Chéron A, Barloy-Hubler F: Identification of chromosomal alpha-proteobacterial small RNAs by comparative genome analysis and detection in Sinorhizobium meliloti strain I02I. BMC Genomics 2007, 8:467.

24. Kaneko T, Nakajima N, Okamoto S, Suzuki I, Tanabe Y, Tamaoki M, Nakamura Y, Kasai F, Watanabe A, Kawashima K, et al.: Complete genomic structure of the bloom-forming toxic cyanobacterium Microcystis aeruginosa NIES-843. DNA Res 2007, 14(6):247-256.

25. Prediction of ncRNAs in model cyanobacteria website [http:l /www.cyanolab.de/paper/suppl/syncomp]

26. Washietl S, Hofacker IL, Stadler PF: Fast and reliable prediction of noncoding RNAs. Proc Natl Acad Sci USA 2005, I02:2454-2459.

27. Griffiths-Jones S, Moxon S, Marshall M, Khanna A, Eddy SR, Bateman A: Rfam: annotating non-coding RNAs in complete genomes. Nucleic Acids Res 2005, 33:D I I - I 24.

28. Kingsford CL, Ayanbule K, Salzberg SL: Rapid, accurate, computational discovery of Rho-independent transcription terminators illuminates their relationship to DNA uptake. Genome Biol 2007, 8:R22.

29. Barrick JE, Corbino KA, Winkler WC, Nahvi A, Mandal M, Collins J, Lee M, Roth A, Sudarsan N, Jona I, et al.: New RNA motifs suggest an expanded scope for riboswitches in bacterial genetic control. Proc Natl Acad Sci USA 2004, I 0 I:642 I-6426.

30. Rodionov DA, Vitreschak AG, Mironov AA, Gelfand MS: Comparative genomics of thiamin biosynthesis in procaryotes. New genes and regulatory mechanisms. J Biol Chem 2002, 277:48949-48959.

31. Zengel JM, Lindahl L: Diverse mechanisms for regulating ribosomal protein synthesis in Escherichia coli. Prog Nucleic Acid Res Mol Biol 1994, 47:33I-370.

32. Oliveros JC: VENNY. An interactive tool for comparing lists with Venn diagrams. [http://bioinfogp.cnb.csic.es/tools/venny/ index.html].

33. Nakamoto $\mathrm{H}$, Suzuki M, Kojima K: Targeted inactivation of the hrcA repressor gene in cyanobacteria. FEBS Lett 2003, 549:57-62.

34. Glatz A, Horvath I, Varvasovszki V, Kovacs E, Torok Z, Vigh L: Chaperonin genes of the Synechocystis PCC 6803 are differentially regulated under light-dark transition during heat stress. Biochem Biophys Res Commun 1997, 239:291-297.

35. Hsu LM, Zagorski J, Wang Z, Fournier MJ: Escherichia coli 6S RNA gene is part of a dual-function transcription unit. J Bacteriol 1985, 161:1162-1170.

36. Kim KS, Lee Y: Regulation of 6S RNA biogenesis by switching utilization of both $\mathbf{s}$ factors and endoribonucleases. Nucleic Acids Res 2004, 32:6057-6068.

37. Hershberg R, Altuvia S, Margalit H: A survey of small RNA-encoding genes in Escherichia coli. Nucleic Acids Res 2003, 31: I8I3-1820.

38. Hermann T, Patel DJ: RNA bulges as architectural and recognition motifs. Structure 2000, 8:R47-R54.

39. Babitzke P, Romeo T: CsrB sRNA family: sequestration of RNA-binding regulatory proteins. Curr Opin Microbiol 2007. I0:156-163.

40. Valverde C, Lindell M, Wagner EG, Haas D: A repeated GGA motif is critical for the activity and stability of the riboregulator RsmY of Pseudomonas fluorescens. J Biol Chem 2004, 279:25066-25074.

41. Overview on repeat elements in model cyanobacteria [http:/ /www.cyanolab.de/paper/suppl/syncomp/repeat cluster.html]

42. Rippka R, Deruelles J, B W], Herdmann M, Stanier RY: Generic assignments, strain histories and properties of pure cultures of cyanobacteria. J Gen Microbiol 1979, II I: I-6I.

43. Kunert A, Hagemann M, Erdmann N: Construction of promoter probe vectors for Synechocystis sp. PCC 6803 using the light- 
emitting reporter systems Gfp and LuxAB. Journal of Microbiological Methods 2000, 41: 185-194.

44. Dienst D, Dühring U, Mollenkopf H, Vogel J, Golecki J, Hess WR, Wilde A: The RNA chaperone $\mathbf{H f q}$ is essential for cell motility of the cyanobacterium Synechocystis PCC 6803. Microbiology 2008, 154:3|34-3|43.

45. Genbank [http://www.ncbi.nlm.nih.gov/genomes/lproks.cgi]

46. Kurtz S: The Vmatch large scale sequence analysis software. [http://www.vmatch.de].

47. Thompson JD, Higgins DG, Gibson TJ: CLUSTAL W: improving the sensitivity of progressive multiple sequence alignment through sequence weighting, positions-specific gap penalties and weight matrix choice. Nucleic Acids Res 1994, 22:4673-4680.

48. Voss B: Structural analysis of aligned RNAs. Nucleic Acids Res 2006, 34:547I-548I.

49. Griffiths-Jones S: RALEE - RNA ALignment editor in Emacs. Bioinformatics 2005, 21:257-259.

50. Hofacker IL, Fekete M, Stadler PF: Secondary structure prediction for aligned RNA sequences. J Mol Biol 2002, 319(5): 1059-1066.

Publish with Bio Med Central and every scientist can read your work free of charge

"BioMed Central will be the most significant development for disseminating the results of biomedical research in our lifetime. "

Sir Paul Nurse, Cancer Research UK

Your research papers will be:

- available free of charge to the entire biomedical community

- peer reviewed and published immediately upon acceptance

- cited in PubMed and archived on PubMed Central

- yours - you keep the copyright

Submit your manuscript here:

http://www.biomedcentral.com/info/publishing_adv.asp
BiolMedcentral 